\title{
Investigations of Quantum Cascade Lasers for Free Space Optics Operating at the Wavelength Range of 8-12 $\mu \mathrm{m}$
}

\author{
M. Nowakowski, M. Gutowska, D. Szabra, J. MikoŁajczyk, J. Wojtas
}

AND Z. BIELECKI

Institute of Optoelectronics, Military University of Technology, S. Kaliskiego 2, 00-908 Warsaw, Poland

The paper presents analysis and preliminary investigations of quantum cascade lasers for free space optics. The lasers radiate in the long wavelength IR spectral range $(8-12 \mu \mathrm{m})$. Because of lower effects of radiation scattering than in the case of $1.5 \mu \mathrm{m}$ free space optics, better transmission range can be obtained. The main task of the work was experimental investigation of quantum cascade lasers taking into consideration free space optics applications. In the research, quantum cascade lasers operating in both pulse and continuous wave modes were used. The lasers spectra as well as the operation characteristics (e.g. voltage vs. current) were measured. In conclusion, the quantum cascade lasers parameters for free space optics system applications are summarized.

PACS: 42.62.-b, 01.50.Pa

\section{Introduction}

For more than three decades dynamic development of the free space optics (FSO) communication systems can be observed [1-6]. Analysis and literature studies show that better communication ranges can be obtained using FSO systems working in the 3rd transmission window of the atmosphere [7-9]. In comparison to other windows, the lower effect of radiation scattering is noticed in this spectral range [10]. This is very evident especially in the case of bad weather conditions (hazy day) or air pollution $[11,12]$. For many years the main problem of the FSO technology development operating at the wavelength range of 8-12 $\mu \mathrm{m}$ was the lack of suitable radiation sources. The $\mathrm{CO}_{2}$ lasers required complicated power supply units and they were large, heavy and expensive. The development of materials technology and other related areas allows to product new sources of long wavelength radiation - quantum cascade (QC) lasers [13-16]. The QC lasers are characterized by better practical features for FSO applications. The structures of QC lasers are continually developed [17]. Researches are concentrated on increase in optical power, repetition rate, and duty cycle of the laser pulses in the room temperature operation.

\section{Quantum cascade lasers}

In the case of applications the QC lasers in the FSO transmitter, ranges of spectrum, peak powers, repetition rates and duty cycles of the radiation pulses should be taken into consideration. Market analysis shows that there are several models of cascade lasers. The lasers are constructed to operate in pulsed mode, continuous mode, or both in continuous and pulsed mode. Figure 1 shows some available types of QC lasers.

The main parameters of the lasers operation are determined by characteristics of both current vs. voltage and

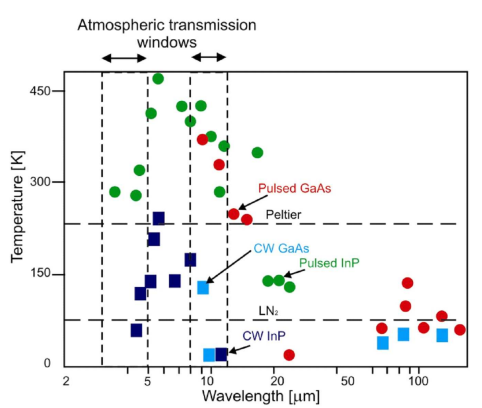

Fig. 1. Commercially available QCL lasers [18].

current vs. radiation power. Some characteristics of QCL laser are presented in Fig. 2.

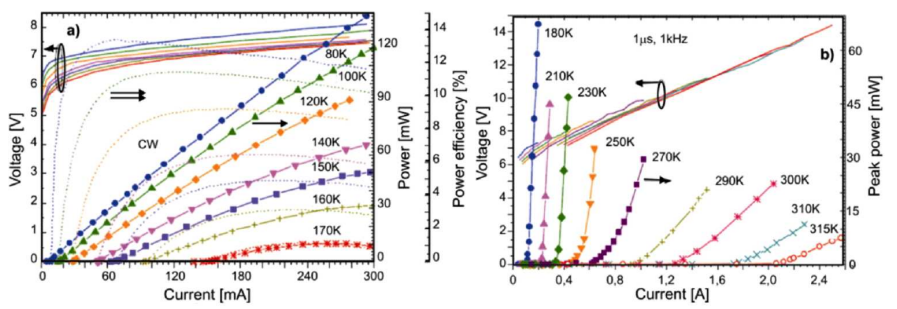

Fig. 2. QCL laser characteristics for CW mode (a) and pulse mode (b) [18].

It can be seen that the value of laser power depends on current and operation temperature.

The pulsed QC lasers are characterized by high value of both radiation power and threshold current. But lasers available today in the most cases can only work with a repetition rate less than $1 \mathrm{MHz}$ and duty cycle (DC) of about $1-5 \%$. These parameters significantly limit the bandwidth of optical data link and necessitate the use of specific transmission algorithm codes. The investigations 
performed with research group from Alpes Lasers company showed that offered pulsed lasers were able to work with a repetition rate of $5 \mathrm{MHz}(\mathrm{DC}<1 \%)$. However, at high repetition rates $(>2 \mathrm{MHz})$ the peak power of the emitted pulses was changed nonlinear. This phenomenon could significantly decrease in the data link range.

Continuous wave QC lasers are another step in the development of some science and technology fields. During operation, bias current flows through the laser structure all the time. Therefore, a large amount of heat is produced. The very efficient cooling system is required to control the laser temperature. QCL technology development makes it possible to produce CW lasers operating at the room temperature. These lasers are characterized by single mode structure of radiation. Parameters of laser working operation significantly influence on the spectra shape operational characteristics (Fig. 3).

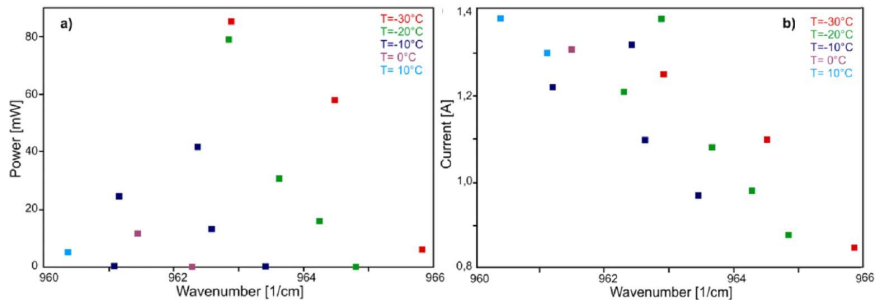

Fig. 3. Spectral characteristics of the CW laser for different temperatures: power (a) and current (b) [18].

Figure 3 shows that the growth in current increases the wavelength range of the radiation. During decrease in temperature, the average power is increased.

Alpes Lasers company is one of the leading QC lasers developers. The company designs and produces so-called high-power Fabry-Perot lasers. The lasers are able to operate in either pulsed or continuous mode without any limit on the pulse duration. In Fig. 4 some high-power Fabry-Perot lasers are compared.

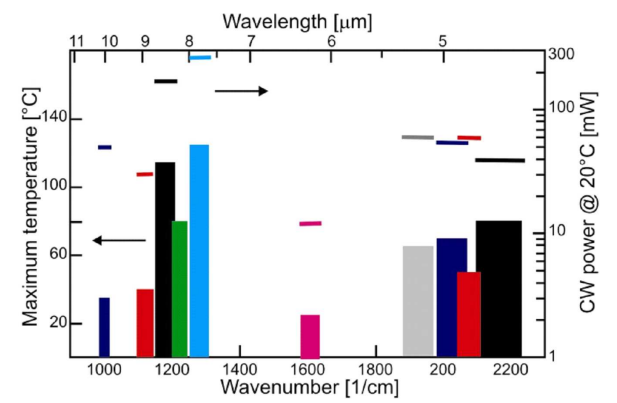

Fig. 4. Comparison of some high power QCL lasers [18].

\section{QC lasers research}

In the case of application of QC laser to FSO transmitter, special attention should be paid to the range of wavelengths of the emitted radiation, the ability to operate with high repetition (unlimited duty cycle of pulses) and the possibility of obtaining a maximum power of this radiation. That is why the main task of the research was to determine the virtues of the selected QC lasers. Based on data submitted by the manufacturers of lasers it was found that the very promising radiation sources would be a high-power laser operating in both continuous mode and pulse sbcw2968 and sbcw3252 models. For the power supply and control of the lasers, the special driver system produced by Alpes Lasers was applied (Fig. 5). The system consists of a laser housings, laser control module and controller of thermoelectric cooler modules. The cooling unit stabilizes the lasers temperature in the range of $-40^{\circ}$ to $+80^{\circ}$.

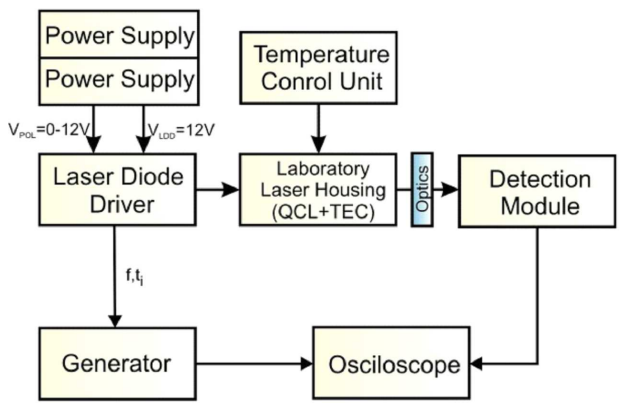

Fig. 5. Driver system produced by Alpes Lasers applied to laboratory setup.

\subsection{Investigation of laser pulse shape}

The research of laser pulse shape was carried out using the QC laser driver system, detection module produced by Vigo S.A PVI series [19-23] and oscilloscope Tektronix series 4000. In Fig. 6 the shapes of laser pulses for different values of the voltage fed to light detecting diode (LDD) amplitude are presented. Measurements were made for pulse repetition rate of $2 \mathrm{MHz}$, pulse duration of $50 \mathrm{~ns}$ and stabilization temperature of about $10^{\circ} \mathrm{C}$. It is noticed that the value of threshold voltage was about $8.5 \mathrm{~V}$. With increasing voltage value, shape of laser pulses also changes.

At the voltage value of $9.5 \mathrm{~V}$, two pulses can be observed. Further increase in the voltage to the value of above $11 \mathrm{~V}$ results to a quasi-rectangular pulse. The shape changes of laser pulses are caused by a change in temperature and electrical properties of the laser. Due to the short pulse durations compared with a time constant of the cooling system, dynamic changes in the working point of the laser can be observed. Operating at the control voltage near the threshold value, the blanking pulse can be observed (Fig. 6b,c). If detection module is fast enough, the double bits can be registered. That is why for communication systems this phenomenon is not desirable because the value of bit error rate could be increased. The elimination of the described errors requires a number of additional studies (software or hardware). 


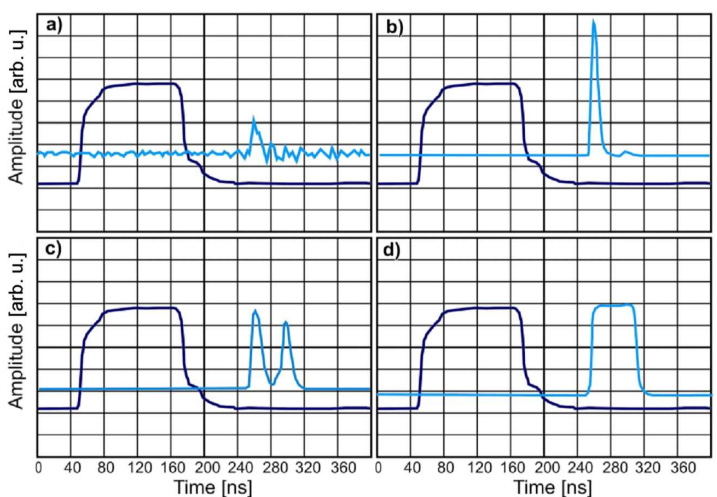

Fig. 6. Shapes of laser pulse (cyan line) for different values of the voltage fed to LDD amplitude (blue dot line): $V=7 \mathrm{~V}, I=75 \mathrm{~mA}(\mathrm{a}), V=8.5 \mathrm{~V}, I=$ $101 \mathrm{~mA}$ (b), $V=9.5 \mathrm{~V}, I=119 \mathrm{~mA},(\mathrm{c}) V=11 \mathrm{~V}$, $I=164 \mathrm{~mA}(\mathrm{~d})$.

\subsection{Power-voltage characteristics}

During measurements of the laser power characteristics, a Standa power meter with silicon head 11XLP12-1S-H2-D0, Stanford Research DG645 generator, Agilent E3631A and E3612A power supplies were used (Fig. 7).

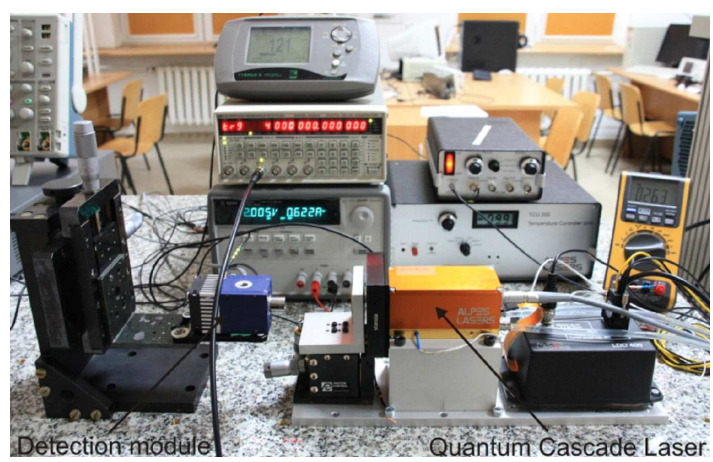

Fig. 7. Laboratory setup to measure optical power of the lasers.

The characteristics of measured average optical power of the lasers versus control voltage for several values of temperature and duty cycle are shown in Figs. 8 and 9. Basing on the test results, it can be observed that when the duty cycle increases, the average power of radiation increases. This growth is a nonlinear relationship with values of duty cycle. Simultaneously increase in temperature causes a drop in the laser average power of radiation, an increase of the threshold voltage and change of the slope of the current versus optical power characteristics. For this reason, the important issue using QC lasers is to provide a suitable (efficient and fast) cooling system.

\subsection{Current-voltage characteristics}

Investigations of the current-voltage characteristics were performed for different values of operating tempera-
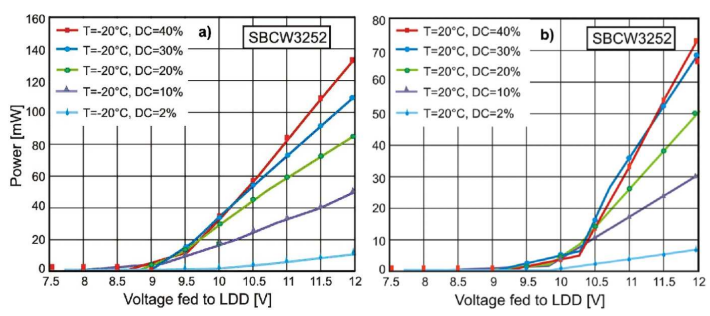

Fig. 8. Average power vs. voltage fed to LDD of sbcw3252 laser for different values of temperature and pulse duty cycle.

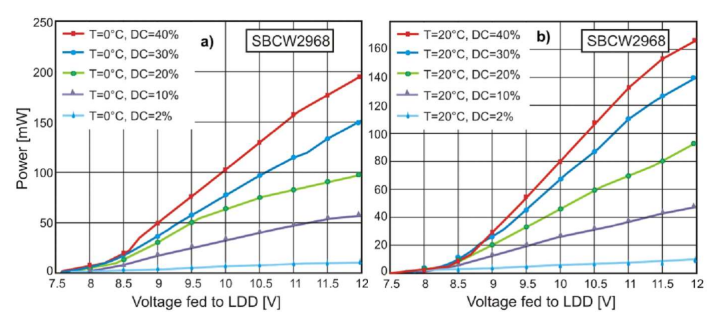

Fig. 9. Average power vs. voltage fed to LDD of sbcw2968 laser for different values of temperature and pulse duty cycle.

tures and pulse duty cycle (at the lab setup presented in Fig. 7). Figure 10 shows the determined current-voltage characteristics of the tested lasers. Duty cycle was tuned by changing value of repetition rates for constant value of pulse duration (100 ns). It is noticed that when the value of duty cycle is increasing, the control current also increases. This growth is a nonlinear relationship with increasing values of duty cycle. However, the increase in operating temperature does not cause significant changes in value of current.

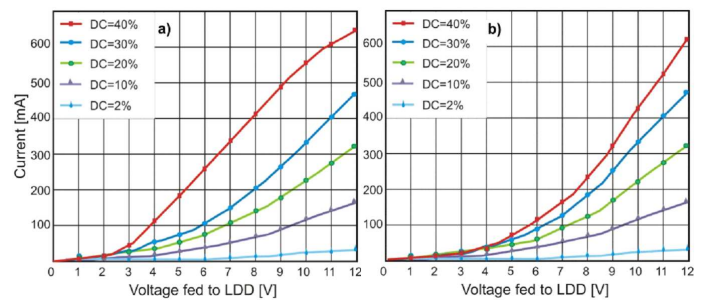

Fig. 10. Current vs. voltage fed to LDD of sbcw3252 (a) and scbw2968 (b) lasers for different values of pulse duty cycle.

\subsection{Spectrum measurements}

Investigations of the lasers spectrum were performed using a spectrometer Horiba model iHR320. Measurements were made for repetition rate of $4 \mathrm{MHz}$. The registered spectra are shown in Fig. 11. It is shown that the both lasers emit radiations with multimode structures. It results directly from the lasers construction and features of the Fabry-Perot resonator. However, the value 
changes in temperature and laser current generally influence on spectral range and shape of the emitted radiation.

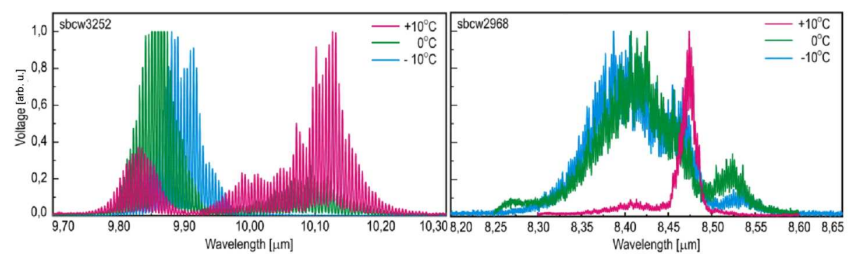

Fig. 11. Registered spectra of QC lasers.

According to the manufacturer's specifications both lasers were optimized in long wavelength IR (LWIR) spectral range: sbcw3252 for value of $10 \mu \mathrm{m}$ and sbcw2968 for value of $8.5 \mu \mathrm{m}$. The obtained spectra for two lasers are comparable with catalogue data.

\section{Conclusions}

Researches were conducted to determine the virtues of sbcw3252 laser and sbcw2968 laser, taking into account the possibility of its use in the free space optical link. The investigations included:

- determination of the influence of control voltage on the optical laser pulse shape,

- measuring changes in the characteristics of the average optical power as a function of voltage, temperature and duty cycle laser pulses,

- designation of the spectral characteristics.

The study shows that lasers sbcw3252 and sbcw2968 from Alpes Lasers can be successfully used in the FSO link.

\section{Acknowledgments}

The authors would like to thank the Ministry of Science and Higher Education for their support in the field. The article was developed under research grant no. O R00 00 8606 .

\section{References}

[1] H. Manor, A. Argon, Appl. Opt. 42, 21 (2003).

[2] M. Achour, "Free space optics builds invisible selection: $10 \mu \mathrm{m}$ versus shorter wavelengths", http://www.ulmtech.com .

[3] E. Leitgeb, T. Plank, M.S. Awan, P. Brandl, W. Popoola, Z. Ghassemlooy, F. Ozek, M. Wittig, in: Proc. of ICTON, Microwave Association, Paris 2010, p. 37.

[4] J.C. Juarez, IEEE Comm. Mag. 121, 46 (2006).
[5] Z. Bielecki, W. Kołosowski, J. Mikołajczyk, M. Nowakowski, in: PIERS, Cambridge (USA), 2008, Conf. Proc., E-M Academy 2008, p. 95, www . emacademy . org .

[6] R. Martini, in: Proc. 5th QCL Workshop, Freiburg (Germany), Fraunhofer IPM, Freiburg 2004, p. 311.

[7] A. Rogalski, Z. Bielecki, in: Handbook of Optoelectronics, Vol. I, Taylor \& Francis, New York 2006.

[8] X. Liu, in: Proc. Int. Conf. on Communications, Hong-Kong, IEEE 2007, p. 2457.

[9] Z. Bielecki, J. Mikołajczyk, M. Nowakowski, J. Wojtas, Proc. Electrotechn. Instit. 234, 135 (2008) (in Polish).

[10] M. Aharonovich, S. Arnon, J. Opt. Soc. Am. 22, 1646 (2005).

[11] B.R. Strickland, M.J. Lavan, E. Woodbridge, V. Chan, Appl. Opt. 38, 424 (1999).

[12] S.S. Mohammad, B. Flecker, E. Leitgeb, M. Gebhart, J. Opt. Eng. 46, 066001 (2007).

[13] J. Faist, C. Gmachl, F. Capasso, C. Sirtori, D.L. Sivco, J.N. Baillargeon, A.Y. Cho, Science 264, 553 (1994).

[14] R. Pabjańczyk, M. Sarzała, W. Wasiak, M. Bugajski, Elektronika 21, 30 (2009) (in Polish).

[15] F. Capasso, R. Paiella, R. Martini, R. Colombelli, C. Gmachl, T.L. Mayers, M.S. Taubman, R.M. Williams, C.G. Bethea, K. Unterrainer, H.Y. Hwang, D.L. Sicvo, A.Y. Cho, A.M. Sergent, H.C. Liu, E.A. Whittaker, IEEE J. Quantum Electron. QE 38, 511 (2002).

[16] J. Faist, F. Capasso, C. Sirtori, D.L. Sivco, J.N. Baillargeon, A. Hutchinson, S.N. Chu, A.Y. Cho, Appl. Phys. Lett 68, 3680 (1996).

[17] M. Bugajski, K. Kisiel, A. Szerling, J. Kubacka-Traczyk, I. Sankowska, P. Karbownik, A. Trajnerowicz, E. Pruszyńska-Karbownik, K. Pierściński, D. Pierścińska, Bull. Pol. Acad. Sci. Techn. Sci. 58, 471 (2010).

[18] www.alpeslasers.com .

[19] M. Gutowska, W. Gawron, M. Nowakowski, Z. Bielecki, J. Mikołajczyk, D. Szabra, Photon. Lett. Poland 2, 88 (2010).

[20] P. Struk, T. Pustelny, K. Gut, K. Golaszewska, E. Kaminska, M. Ekielski, I. Pasternak, E. Łusakowska, A. Piotrowska, Acta Phys. Pol. A 116, 414 (2009).

[21] M. Gutowska, M. Nowakowski, Z. Bielecki, J. Mikołajczyk, D. Szabra, R. Paliwoda, M. Pędzińska, J. Pawluczyk, W. Gawron, in: Proc. ELTE IMAPS, Wrocław University of Technology, Wrocław (Poland) 2010, p. 231.

[22] T. Pustelny, I. Zielonka, C. Tyszkiewicz, P. Karasinski, B. Pustelny, Opto-electron. Rev. 14, 161 (2006).

[23] M. Gutowska, M. Nowakowski, Z. Bielecki, J. Mikołajczyk, D. Szabra, R. Paliwoda, M. Pędzińska, J. Pawluczyk, W. Gawron, Acta Phys. Pol. A 118, $1143(2010)$. 